\title{
Impact of Heterogeneity and Lattice Bond Strength on DNA Triangle Crystal Growth
}

Evi Stahl ${ }^{1}$, Florian Praetorius ${ }^{1}$, Carina C. de Oliveira Mann², Karl-Peter Hopfner ${ }^{2}$, Hendrik Dietz $^{1 \#}$

${ }^{1}$ Physik Department \& Institute for Advanced Study, Technische Universität München, Am Coulombwall 4a, 85748 Garching near Munich, Germany

${ }^{2}$ Department of Biochemistry and Gene Center, Ludwig-Maximilians-Universität, FeodorLynen-Str. 25, 81377 Munich, Germany

\section{SUPPLEMENTARY INFORMATION}

Note S1

Note S2

Figure $\mathrm{S} 1$

Figure S2

Figure S3

Figures S4 - S9

Figure S4

Figure S5

Figure S6

Figure S7

Figure S8

Figure S9

Figure $\mathrm{S} 10$

Figure S11

Table S1

References
Sample preparation

Crystallization in the presence of pseudo-defective triangle strands

Crystal scoring

Diffraction patterns

Crystallization of triangles with fluorescent labels

Triangle assembly and fluorescence tracing

Truncations

Mismatches

Base pair insertions

Base pair deletions

Insertion bulges

Deletion bulges

Extended version of Figure 2

Sequence variants sorted according to nearest neighbor parameters

List of oligonucleotide sequences 


\section{Note S1: Sample preparation}

Triangle strands ( $\mathrm{L}, \mathrm{M}$, and $\mathrm{S}$ ) were combined to an "original" pool containing $30 \mu \mathrm{M} \mathrm{M}$ strand, $90 \mu \mathrm{M} L$ strand, and $90 \mu \mathrm{M}$ S strand. This reaction mixture was modified or combined with different components as described in the following.

Figure 1 b, c: The "original" pool was further diluted in water prior to setting up crystallization droplets, to obtain various concentrations as indicated.

Figure 1 d, e: Three titration series for each strand (L, $M, S)$ were prepared, in which the concentration of the particular strand in the "original" pool was adjusted according to the indicated stoichiometric factors (0.3-2).

Figures 2, 3: A "modified" pool was prepared with an M strand concentration of $30 \mu \mathrm{M}$ as described for the "original" pool, but one of the original strands (L, M, or S) was replaced by one (single strand modifications) or two (double strand modifications) corresponding modified strands at the according concentrations (see Table S1 for details). "Modified" and "original" strand pools were mixed to a sample series containing $0,1,10,50,90$, and $99 \%(\mathrm{v} / \mathrm{v})$ "modified" pool and used in crystallization assays as described above.

Figures S4-S10: For fluorescence tracing experiments, "modified labeled" pools were prepared as described for figs 2 and 3 , but with strands that contained a fluorescent label in addition to the particular modification of interest (see Table S1 for details). "Modified labeled" and "original" pools were mixed to a sample series containing 10, 50, and $90 \%(\mathrm{v} / \mathrm{v})$ "modified labeled" pool and used in crystallization assays as described above.

Figure $4 \mathrm{c}$, d: The "original" pool was mixed with a "random" pool containing 179 different oligonucleotides, each at a concentration of $560 \mathrm{nM}$ (total nucleic acid concentration in the "random" pool was $100 \mu \mathrm{M}$ ). "Random" and "original" pools were mixed to contain 50 and $90 \%(\mathrm{v} / \mathrm{v})$ "random" pool and used in crystallization assays as described above. The sequences of these oligonucleotides were 42-bases long reverse-complementary segments of the M13-derived single-stranded phage genome p7560 (the full genome would be 180 segments).

Figure 4e: The "original" pool was mixed with a BSA solution (NEB, 10mg/ml) to contain $10 \%(\mathrm{v} / \mathrm{v})$ BSA solution and used in crystallization assays as described above. The BSA solution comprised $\sim 150 \mu \mathrm{M}$ BSA, $20 \mathrm{mM}$ Tris- $\mathrm{HCl}, 100 \mathrm{mM} \mathrm{KCl}, 0.1 \mathrm{mM}$ EDTA, and $50 \%$ glycerol ( $\mathrm{pH}$ 8).

Figure 4f: Crystallization assays for this particular experiment were carried out at buffer conditions derived from the original publication on DNA triangle crystallization (1). Here, we used a sequence variant of the DNA triangle, were the arm regions of two interacting triangles formed an eight-base-long, weak affinity recognition site for the TAL protein used here (see Table S1 for details). Triangle strands were mixed at a stoichiometry of L:M:S = 3:1:3 with an $\mathrm{M}$ strand concentration of $25 \mu \mathrm{M}$ in $20 \mathrm{mM} \mathrm{MgCl}$. The mixture was annealed at a thermal ramp from 65 to $20^{\circ} \mathrm{C}$ with $1^{\circ} \mathrm{C} / \mathrm{min}$ and was subsequently mixed with protein solution. For crystallization assays, $3.3 \mu \mathrm{L}$ of the DNA-protein premix were added to $1.7 \mu \mathrm{L}$ buffer to obtain droplets comprising $6 \mu \mathrm{M}$ DNA triangle, $2 \mu \mathrm{M}$ TAL protein, $25 \mathrm{mM}$ Tris- $\mathrm{HCl}, 50 \mathrm{mM}$ magnesium acetate, $30 \mathrm{mM}$ sodium cacodylate, $5 \mathrm{mM}$ magnesium chloride, $50 \mathrm{mM}$ ammonium sulfate, $100 \mathrm{mM}$ sodium chloride, and $4 \mathrm{mM}$ TCEP. The droplets were equilibrated against a reservoir solution containing $1.7 \mathrm{M}$ ammonium sulfate while being annealed at a thermal ramp decreasing from $40{ }^{\circ} \mathrm{C}$ to $20^{\circ} \mathrm{C}$ with $0.2{ }^{\circ} \mathrm{C} / \mathrm{h}$. After ten days of incubation, droplet volumes were reduced to approx. $10 \%$ of the initial volume. 
Figure 5: Triangle strands for the different variants were mixed as described for the "original" pool at an $\mathrm{M}$ strand concentration of $30 \mu \mathrm{M}$ and annealed in $12.5 \mathrm{mM}$ Tris $-\mathrm{HCl}, 10 \mathrm{mM} \mathrm{MgCl}$, $\mathrm{pH} 8.5$ from 65 to $20^{\circ} \mathrm{C}$ with $1^{\circ} \mathrm{C} / \mathrm{min}$. Pre-annealed samples were mixed with our minimal buffer to obtain an $\mathrm{M}$ strand concentration of $6 \mu \mathrm{M}$ in $25 \mathrm{mM}$ Tris- $\mathrm{HCl}$ and $20 \mathrm{mM} \mathrm{MgCl}_{2}(\mathrm{pH}$ 8,5). $5 \mu \mathrm{l}$ droplets of the reaction mixtures were distributed on sitting drop crystallization plates with 48 wells, each containing a reservoir solution of $250 \mathrm{mM}$ Tris- $\mathrm{HCl}$ and $200 \mathrm{mM}$ $\mathrm{MgCl}_{2}, \mathrm{pH} 8.5$ (6 drops per sample variant and plate). To account for possibly slower droplet equilibration and growth at $4{ }^{\circ} \mathrm{C}$, the plates were incubated for 21 days at constant temperatures of $4,16,25$, and $37^{\circ} \mathrm{C}$ and at a thermal ramp decreasing from 60 to $20^{\circ} \mathrm{C}$ with a rate of $0.4{ }^{\circ} \mathrm{C} / \mathrm{h}$ followed by incubation at $20^{\circ} \mathrm{C}$. 


\section{Note S2: Crystallization in the presence of pseudo-defective triangle strands.}

Truncations. 3'-end truncations in the $M$ strand affect the core region of the triangle by creating a flexible single-stranded segment. Since the triangle requires one copy of the $M$ strand per triangle object, mixing truncated $\mathrm{M}$ strands with non-truncated $\mathrm{M}$ strands will lead to two triangle species, "perfect" and "pseudo-defective", with a one base pair energetic bias toward the "perfect" triangle species. Single-base truncated M strands were tolerated in the crystallization, and the truncated pseudo-defective species crystallized even by itself. Fourbase 3' truncations in the $M$ strand were also tolerated, but the corresponding pseudodefective species did not crystallize by itself. 3'-end truncations in the $L$ strand will directly affect triangle-triangle interaction sites. Since the triangle requires three copies of the $L$ strand per triangle object, mixing truncated $L$ strands with non-truncated $L$ strands can in principle generate four different triangle species: "perfect" and "pseudo-defective" in one, two, or all three copies of the $L$ strand, with an energetic bias toward the perfect species. Up to $10 \%$ of one or three base truncated $L$ strands were tolerated in the crystallization of the triangle, but higher ratios of truncated versus non-truncated $L$ strands suppressed crystal growth (Figs $2 b$, S4, S10b).

Mismatches. Sequence mismatches in the core region of the triangle affect the thermodynamic stability (2-5), and thus potentially also the rigidity of the triangle. For up to four mismatches in the $M$ strands, the corresponding pseudo-defective triangle species crystallized by themselves. Triangles with $L$ strands with one mismatch crystallized by themselves, $L$ strands with two mismatches were still tolerated in the triangle crystallization (Figs. 2c, S5, S10c).

Base pair insertions and deletions. Double-strand insertions or deletions in the triangle will lead to deviations from the original triangle shape through helix lengthening or shortening, and underwinding or overwinding, respectively, which could affect the compatibility of the such altered triangles with the crystal lattice. All variants with base pair insertions that we tested assembled into triangles and were also tolerated in the crystallization assay. The variants having base pair insertions in the core region crystallized by themselves, while the variant with insertions in the arm region did not; yet the strands from this species were integrated into the crystal lattice according to fluorescent tracing experiments (Figs 2d, S6, S10d). Base pair deletions in the core region were tolerated in the crystallization. Crystals grown in the presence of $10 \%$ of a species with base pair deletions in the arms region were cubic and diffracted up to $5.0 \AA$. However, at $50 \%$ the crystals became rod-shaped and diffracted worse (7.3 $\AA$ ) (Figs S2, 2d, S7, S10d). Overall, our data indicates that the triangle crystallization is robust to the significant geometrical deformations that are caused by base pair insertions and deletions. This finding may hint at an inherent flexibility of the triangle that can compensate for under or over twisting in addition to helix lengthening or shortening in the context of the crystal lattice.

Insertion Bulges. Another type of structural modifications that we tested were single-strand bulges, which destabilize (2) and potentially alter the geometry $(6,7)$ of double-helical DNA domains. For mixtures containing also one or two one-base insertion bulges in the $M$ strand the crystals were indistinguishable from those formed by pure "perfect" triangles (Fig 2e, S10e). X-ray diffraction experiments with crystals formed in a 1:1 mixture of unmodified $M$ strands versus $M$ strands with two one-base long bulges indicated reflections up to $5.3 \AA$ (Fig. S2). For three and five-base long bulges in the $M$ strand a second, spherule- or rodshaped crystal species appeared when the ratio of modified strand over original $\mathrm{M}$ strand was larger than 1 (Fig. 3 lists the 3x3- variant, see also Figs. 2e, S8, S10e), which we attribute to 
some byproduct that is not the designed triangle. The bulges that we created in the $M$ strand point away from the small inner cavity of the triangles (see Fig. 1a) and are thus unlikely to cause steric conflicts, which may explain why heterogeneity from such bulges is tolerated.

By contrast, when bulges are created in the core in the complementary $L$ strand, the bulges will point into the small inner cavity of the triangles, and could thus cause steric conflicts (see Fig. 1a). Indeed, bulges created by insertions in the $L$ strand had a much stronger effect on the crystallization of the triangle. Somewhat counterintuitively, three-base long bulges in the $\mathrm{L}$ strand were tolerated in the crystallization assay, while one-base bulges in the $\mathrm{L}$ strand inhibited crystal growth (Figs. 2e, S10e). Based on gel electrophoresis we attribute this observation to the fact that $L$ strands with three-base bulges are not integrated into triangles at all (Fig.S8). Hence, a pseudo-defective triangle species does not emerge which could interfere with crystal growth. By contrast, the $L$ strands with one-base bulges do assemble into pseudo-defective triangles with bulges, which negatively influences crystal growth. Single strand bulges in the arms region of the triangle may destabilize the double-helical domains that are important for the lattice interactions in the crystal. One-base insertion bulges in the $L$ strand were tolerated in the crystallization assay, while three-base insertions inhibited crystal growth (Figs. 2e, S8, S10e).

Deletion bulges. Bulges may also be created by deleting bases in a triangle strand, which will create a bulge in the complementary strand. In addition to the effects caused by insertion bulges mentioned above, deletions will additionally affect the overall length and the twist of a double helical domain. One-base bulges in the $S$ strand (created by a one-base deletion in the $L$ strand) were tolerated and lead to overall similar behavior as when we deleted a full base pair in the same region. One-base bulges in the $L$ strand (created by a one-base deletion in the $M$ strand) were tolerated in the triangle crystallization. Again somewhat counterintuitively, three-base bulges in the $M$ strand (created by deletions in the $L$ strand) were tolerated in the crystallization, while one-base bulges inhibited crystal growth. We attribute to the fact that the $L$ strands with three-base deletions are not integrated into triangles and thus do not interfere with crystal growth via pseudo-defective triangle species, while the one-base deletions are integrated and the correspondingly modified triangles negatively affect crystal growth. A three-base bulge in the $S$ strand effectively inhibited crystal growth, presumably by destabilizing a region that is important for lattice interactions (Figs. $2 f$, S9, S10f). 


\begin{tabular}{|c|c|c|c|c|c|c|c|c|c|}
\hline \multicolumn{2}{|c|}{ edge length } & \multicolumn{2}{|c|}{ aspect ratio } & \multicolumn{2}{|c|}{ mono-crystals } & \multicolumn{2}{|c|}{ edge defects } & \multicolumn{2}{|c|}{ face defects } \\
\hline$<10 \mu \mathrm{m}$ & 1 & rods, crumbs & 1 & crowds & 1 & $\begin{array}{c}\text { rough, } \\
\text { incomplete }\end{array}$ & 1 & strong & 1 \\
\hline $10-50 \mu \mathrm{m}$ & 5 & thin platelets & 10 & twins, triplets & 5 & incomplete & 5 & $\operatorname{mix}$ & 5 \\
\hline $50-100 \mu \mathrm{m}$ & 10 & flat squares & 20 & $\operatorname{mix}$ & 10 & $\operatorname{mix}$ & 10 & small & 10 \\
\hline $100-150 \mu \mathrm{m}$ & 15 & cuboid & 25 & single & 15 & $\begin{array}{l}\text { straight, well } \\
\text { defined }\end{array}$ & 20 & no defects & 15 \\
\hline 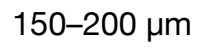 & 18 & cubic-like & 30 & & & & & & \\
\hline$>200 \mu \mathrm{m}$ & 20 & & & & & & & & \\
\hline
\end{tabular}

b

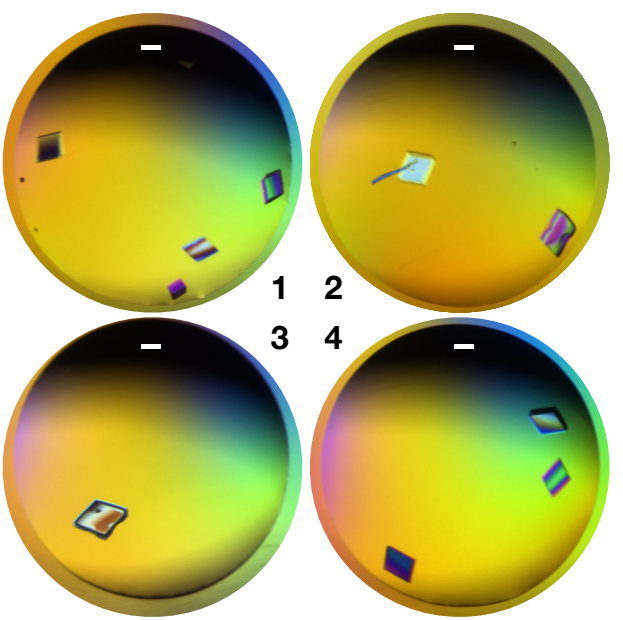

C

\begin{tabular}{l:c:c:c:c:c} 
& $\mathbf{1}$ & $\mathbf{2}$ & $\mathbf{3}$ & $\mathbf{4}$ & \\
edge length & 15 & 18 & 18 & 15 & \\
\hdashline aspect ratio & 25 & 25 & 25 & 25 & \\
\hdashline mono-crystals & 15 & 15 & 15 & 15 & \\
\hdashline edge defects & 20 & 20 & 20 & 20 & \\
\hdashline face defects & 10 & 1 & 5 & 15 & \\
\hline sum & 85 & 79 & 83 & 90 & $84.2 \pm 4.5$
\end{tabular}

Figure S1. Crystal scoring to facilitate comparison of crystal growth in several experiments. (a) Crystals were evaluated according to their macroscopically observable appearance (see b). We regarded aspects such as size and shape of the crystals, and whether they grew in crowds of many, as twins or as single crystals. We also evaluated edge and face defects. Maximum score of 100 is achieved by an ideal rhombohedral crystal with edge length of $\sim 200 \mu \mathrm{m}$. The crystal does not reflect the diffractive qualities of the crystals but rather reflects crystal growth conditions in the droplets. (b) Images of crystals grown in default conditions at an M strand concentration of $60 \mu \mathrm{M}$ in the equilibrated drops. We typically observed cuboid crystals flatter in one dimension, with straight edges and small face defects. Scale bars are $100 \mu \mathrm{m}$. (c) Scoring example shown for the crystal droplets depicted in (b). The resulting crystal score includes an average of four evaluated droplets at identical conditions. Errors give the standard deviation. 
a

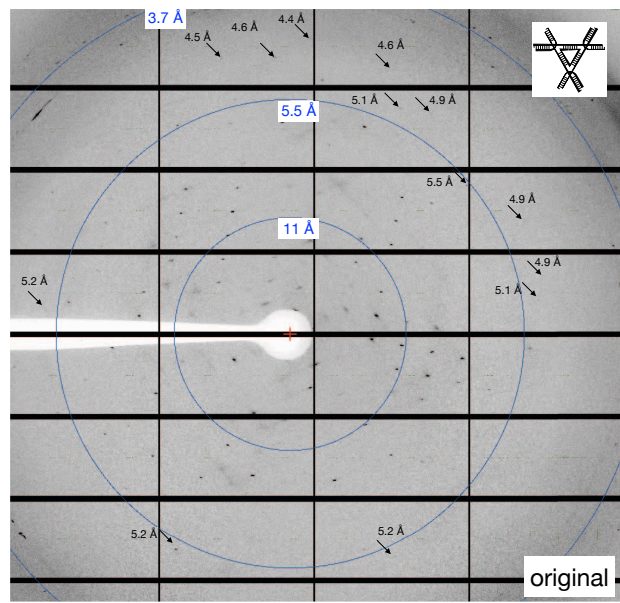

C

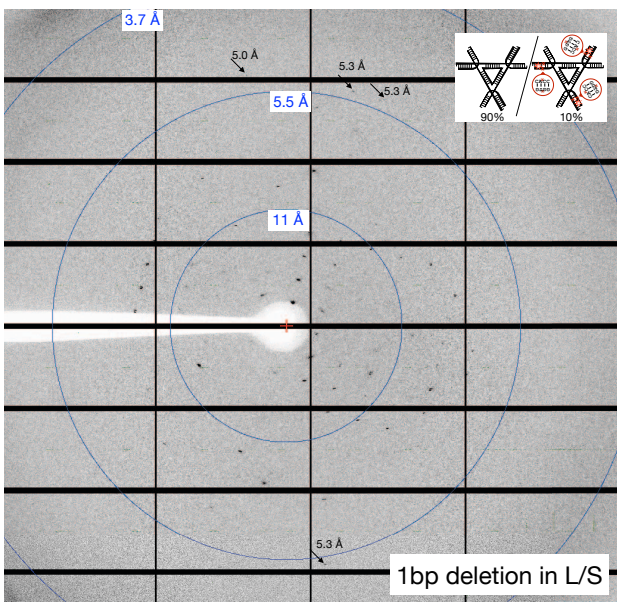

e

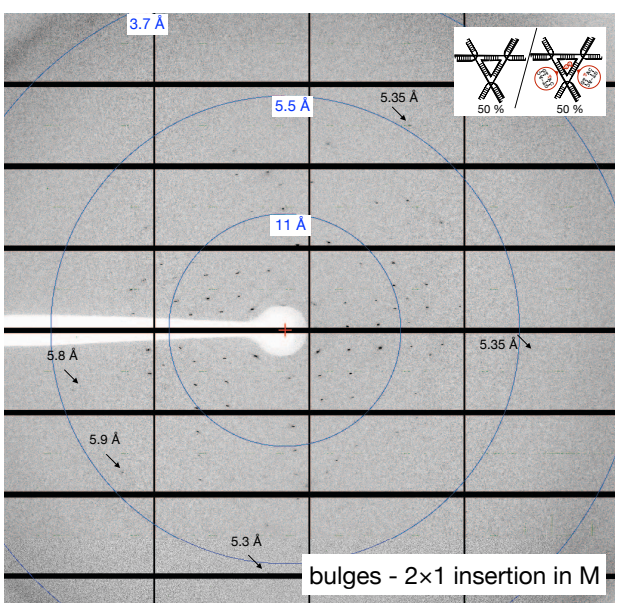

b

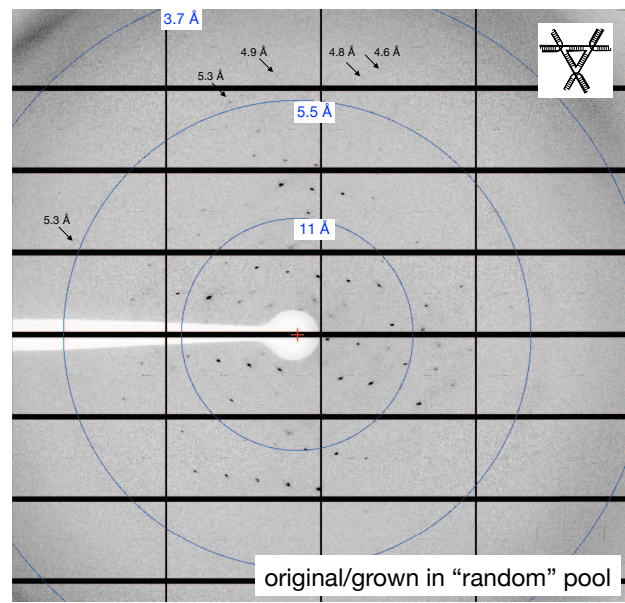

d

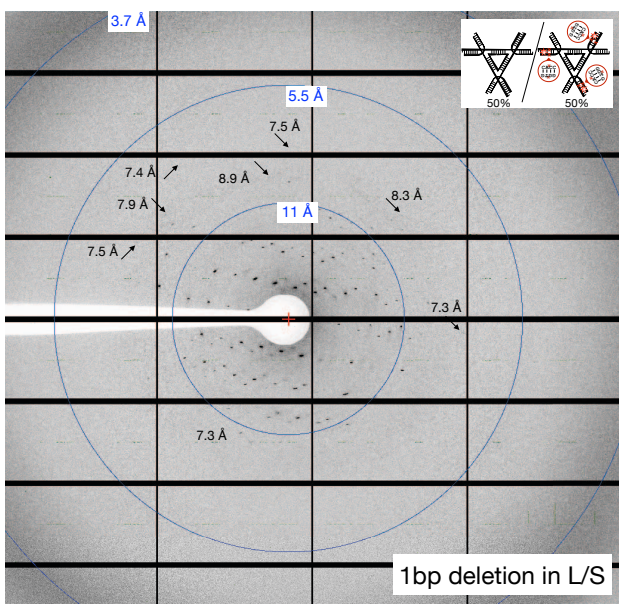

Figure S2. Diffraction patterns of crystals from exemplary triangle variants. DNA triangle crystals showed X-ray diffraction, whether they grew in pure conditions (a), in presence of a "random" pool of competitor oligonucleotides (b) (see also Fig. 4), or in heterogeneous mixtures of original and modified triangle strands (c-e). Schematics in the upper right corner illustrate strand modifications and composition in the reaction mixtures in which the crystals grew. Blue circles in the images indicate the resolution range of observable reflections; arrows point at weak reflections as a guide to the eye. (c, d) Red coloring of the crystals that gave diffraction pattern shown in (c) and (d) indicated incorporation of the modified and labeled S-strand into the crystal lattice (see also Note S2, Fig S7). (e) Triangles with insertions in the M strand assembled and crystallized also by themselves (see Note S2, Figure S8). 


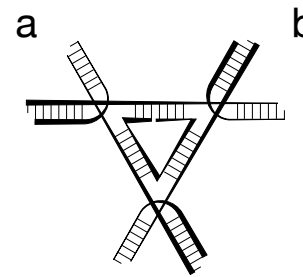

original

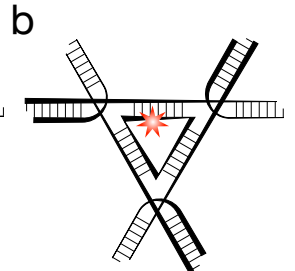

$\mathrm{M}^{*}\left(5^{\prime}\right.$ су 3$)$

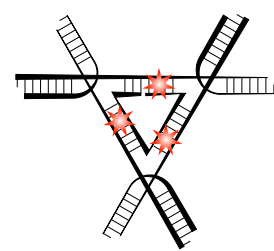

$L^{*}($ atto550)

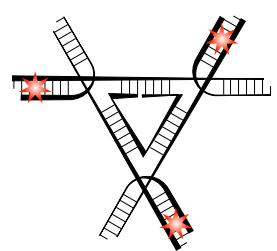

$S^{*}(a t t o 550)$

C

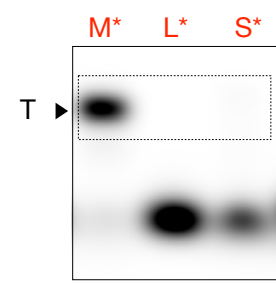

$$
\mathrm{T}
$$

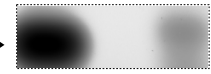

e

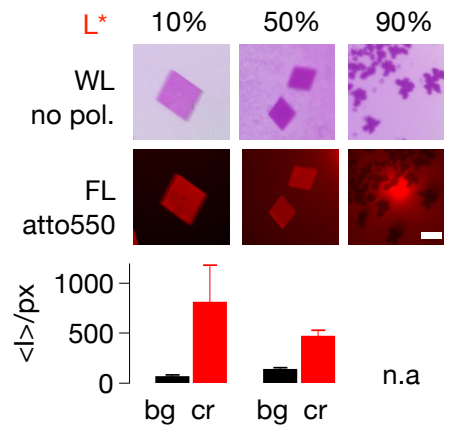

d
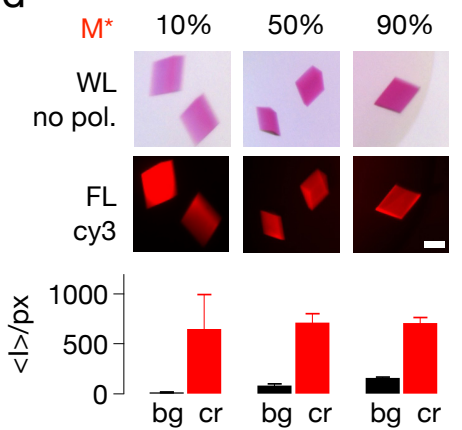

f
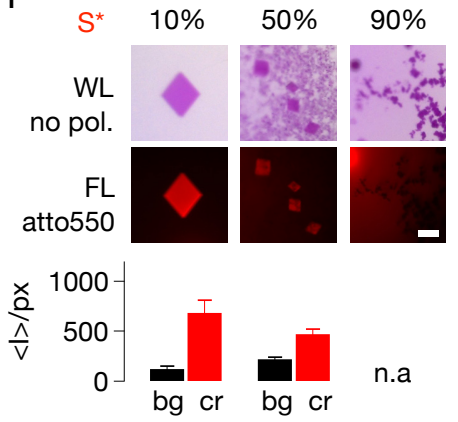

Figure S3. Influence of fluorescent labels on assembly and crystallization of the DNA triangle. (a, b) Schematics illustrate strand topologies of triangles without label (a) and with a fluorophore attached to either of the $M$, $L$ or $S$ strands (b). Labeled $M$ strands $\left(M^{*}\right)$ were chemically conjugated to a cyanine3 fluorophore at their $5^{\prime}$-end. Labeled $L$ and $S$ strands $\left(L^{*}, S^{*}\right)$ contained an amino-modified thymine at the indicated positions that was conjugated to an atto550 fluorophore (see Table S1 for details). (c) Gel electrophoretic analysis of triangle variants in (b) revealed that the presence of internal fluorophore labels significantly impaired the assembly of the strands to triangles, while a label at the 5'-end of the $M$ strand had no negative effects. The boxed gel area is shown again in a strongly contrast enhanced version below the gel. T: triangle band. (d) Typical images of crystals captured under white light (WL, no polarization filters) and fluorescence illumination (FL) confirm that $\mathrm{M}^{\star}$ strands were incorporated into the crystal lattice whether 10,50 or $90 \% \mathrm{M}^{*}$ were present in the mixture of original and labeled triangle strands. (e, f) Images of crystals show the image section at white light illumination (WL, no pol.) and fluorescence illumination (FL). $L^{*}$ and $S^{*}$ strands were incorporated into the crystal lattice despite the given thermodynamic penalty towards triangle assembly. Yet, crystals only grew in presence of more than $50 \%$ original $L$ strands, or $S$ strands, respectively. At higher content $(>50 \%)$ of $L^{*}$ or $S^{*}$ strand, we observed the formation of crystalline aggregates with quenched fluorescence. (d-f, bottom row) Quantification of the fluorescent signal in four identical drops showed a significant increase of fluorescence intensity in the overall area covered by crystals as compared to the droplet background. With increasing content of $\mathrm{M}^{*}, \mathrm{~L}^{*}$ or $\mathrm{S}^{*}$ strands respectively, the average fluorescence intensities in the crystal area did not increase (d) or even decreased (e, f), albeit the color of the crystals became more intense (see WL vs FL). This effect may be due to altered fluorescence properties of the dyes within the crystal lattice. Error bars give standard deviation from the fluorescence quantification in four identical drops. 


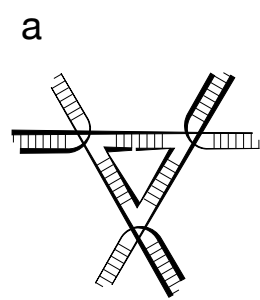

original b

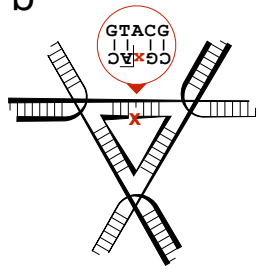

$M^{\prime}\left(3^{\prime}-1\right)$

$\mathrm{M}^{* \prime}\left(3^{\prime}-1\right)$

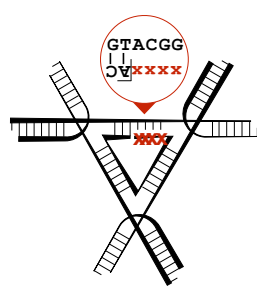

$M^{\prime}\left(3^{\prime}-4\right)$

$M^{* \prime}\left(3^{\prime}-4\right)$

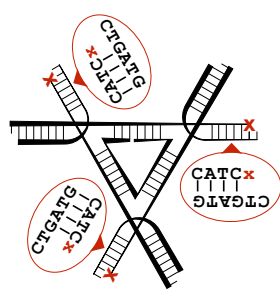

$L^{\prime}\left(3^{\prime}-1\right)$

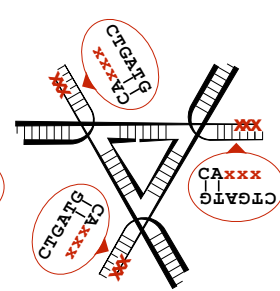

$L^{\prime}\left(3^{\prime}-3\right)$

C

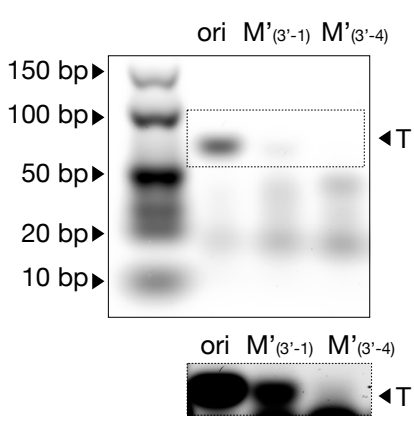

d
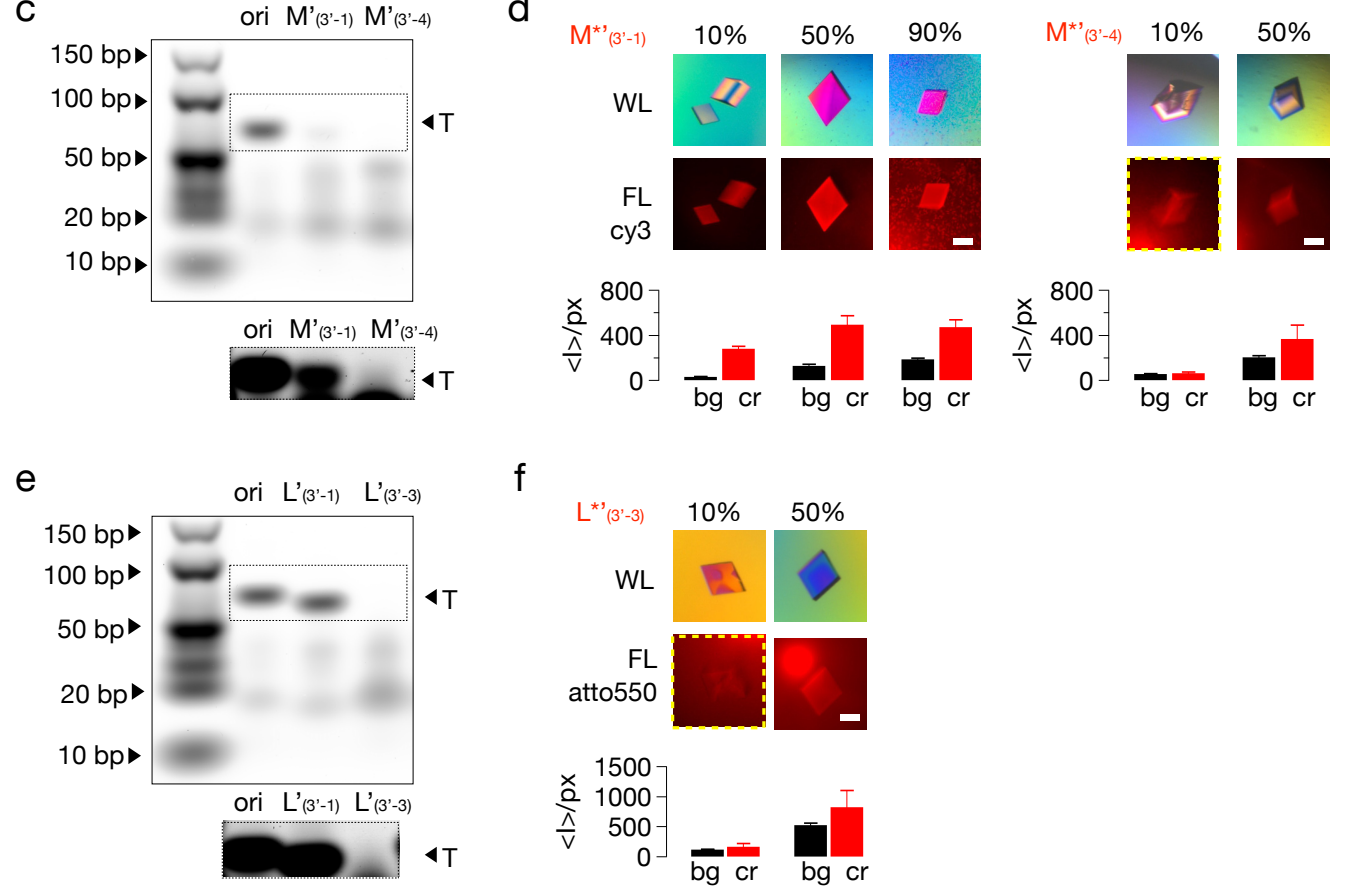

$f$
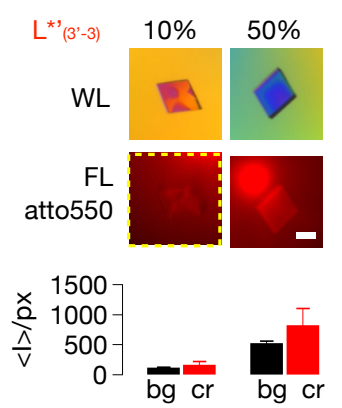

Figure S4. Analysis of triangle assembly and strand incorporation into the crystal lattice for truncated strands. (a, b) Schematics illustrate the composition of the original triangle design (a) and variations (b) designed to contain truncations at the $3^{\prime}$-ends of the $M$-strand ( $M^{\prime}$ ) and of the $L$ strand (L'). (c) Gel electrophoretic analysis of first and second variant listed in (b). Band pattern show that triangles still assemble with truncated $\mathrm{M}$ strands, albeit at significantly reduced yields. The boxed gel area is shown again in a strongly contrast enhanced version below the gel. T: triangle band (d) Exemplary images of crystals from tracing experiments (FL) and overall fluorescence quantification in the droplets (bottom graphs) show strong incorporation of $\mathrm{M}^{{ }^{*}}{ }_{\left(3^{\prime}-1\right)}$ strands and weak incorporation of $\mathrm{M}^{* \prime}{ }_{\left(3^{-}-4\right)}(\mathrm{e})$ Gel electrophoretic analysis of third and fourth variant listed in (b). Band pattern showed that triangles assembled with one-base truncated $L$ strands $\left(L^{\prime}{ }_{\left.\left(3^{\prime}-1\right)\right)}\right.$ but did not form from three-base truncated $L$ strands $\left(\mathrm{L}_{\left(3^{\prime}-3\right)}\right)$. The boxed gel area is shown again in a strongly contrast enhanced version below the gel. T: triangle band (f) Fluorescence quantification from tracing experiments with $L^{*}{ }_{\left(3^{\prime}-3\right)}$ showed that the average fluorescence intensity in the area covered by crystals (cr) was not significantly higher as in the background (bg) for mixtures containing $10 \% \mathrm{~L}^{*^{\prime}}{ }_{\left(3^{\prime}-3\right)}$ (bottom graph). Only strongly contrast enhanced images (yellow frame) indicated a faint fluorescence of the crystals in low background areas, but the majority of labeled $L^{*}$ strands stayed in solution. Yellow frames indicate contrast enhanced images. Error bars in the graphs give standard deviation from four identical drops. Images of crystals show the image section at polarized white light illumination (WL) and fluorescence illumination (FL). (note) Crystals still grew in presence of $50 \% \mathrm{~L}^{*^{\prime}}{ }_{\left(3^{\prime}-3\right)}$ and the modified labeled strands were also incorporated into the crystal lattice. By contrast, crystal growth was inhibited in such mixtures with unlabeled $\mathrm{L}_{\left(3^{\prime}-3\right)}$ strands (see Figs. 2, 3). We attribute this effect to reduced integration of $L^{*}$ ' as compared to $L^{\prime}$, due to the additional thermodynamic penalty towards triangle assembly we saw for strands that carry an internal fluorescent label (Fig S3). This finding points out that pseudo defects such as the three-base truncations have stronger effects on crystallization if they are integrated into triangle-species that can interact with several crystal lattice contacts. 
a

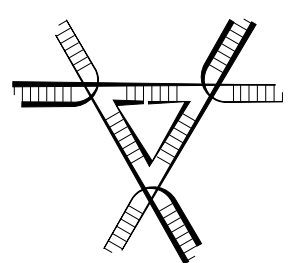

original b

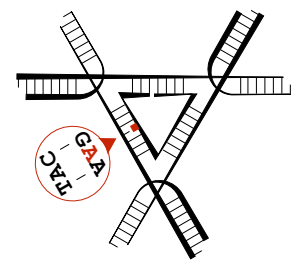

$\diamond \mathrm{M}^{\prime}(1 \mathrm{MM})$

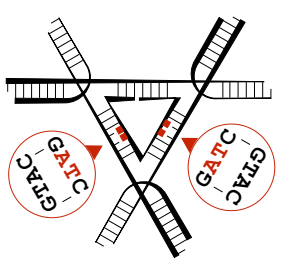

$\square \mathrm{M}^{\prime}(2 \times 2 \mathrm{MM})$ $\mathrm{M}^{*}{ }^{*}(2 \times 2 \mathrm{MM})$

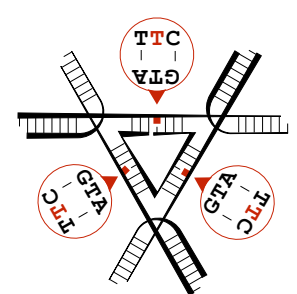

$\triangle \mathrm{L}^{\prime}\left({ }^{1} \mathrm{MM}\right)$

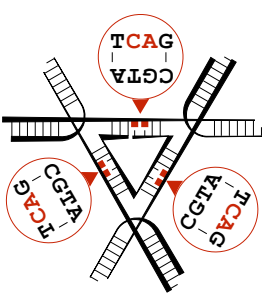

$\boldsymbol{\nabla} \mathrm{L}^{\prime}(2 \mathrm{MM})$
C
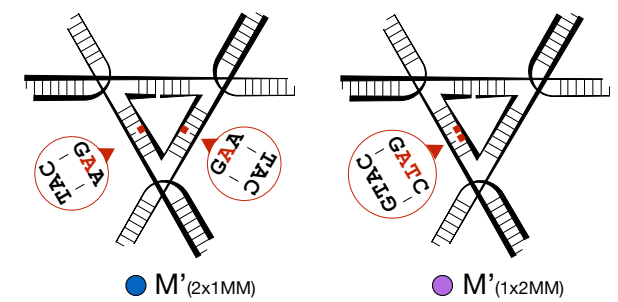

e

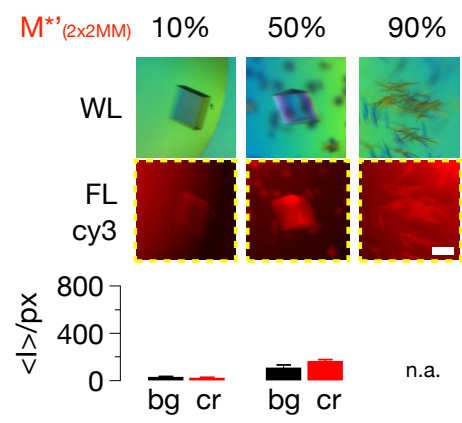

d

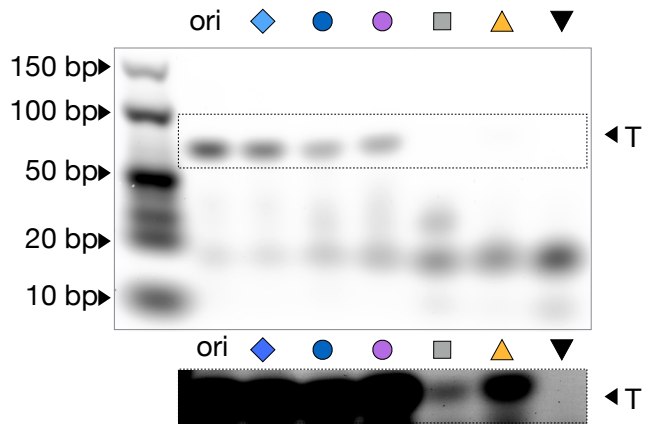

f

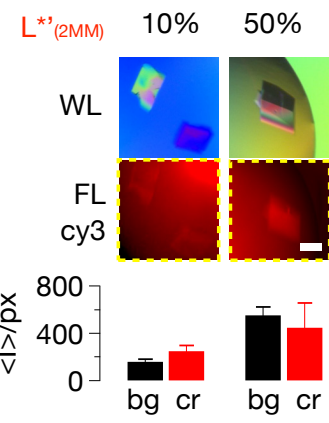

Figure S5. Analysis of triangle assembly and strand incorporation into the crystal lattice for strands that introduce sequence complementarity mismatches. (a-c) Schematics illustrate the composition of the original triangle design (a) and variations designed to contain mismatches in the $M$ strand (M') or in the L strand ( $\left.\mathrm{L}^{\prime}\right)$ (b, c). (d) Gel electrophoretic analysis of triangle variants in (a-c). Band pattern show that triangle assembly yield decreased with increasing number of mismatches and triangles did no longer assemble from $L$ strands with a double mismatch $\left(\mathrm{L}^{\prime}{ }_{(2 \mathrm{MM})}\right)$. The boxed gel area is shown again in a strongly contrast enhanced version below the gel. $T$ indicates the triangle band. (e) Strongly enhanced images of crystals in fluorescence tracing experiments show faint incorporation of $\mathrm{M}^{\star \prime}{ }_{(2 \times 2 \mathrm{MM})}$ into the crystal lattice, but overall quantification of the average fluorescence intensity in the droplets does not give enriched fluorescence in the area covered by crystals (cr) vs. the background area (bg) (bottom graph). We observed that crystalline aggregates had formed at higher concentrations of $\mathrm{M}^{{ }^{*}}{ }_{(2 \times 2 \mathrm{MM})}$. By contrast, mixtures with $99 \%$ unlabeled $\mathrm{M}^{\prime}{ }_{(2 \times 2 \mathrm{MM})}$ strands crystallized also by themselves (Fig 2c, 3). (f) Typical fluorescence images of crystals and overall quantification of the fluorescence indicate a weak integration of $L^{*{ }^{\prime}}{ }_{(2 \mathrm{MM})}$ strand into the crystal lattice, yet a significant fraction of the labeled strand stayed in solution. Results from (d) and (f) suggest that incorporation of $L^{* \prime}{ }_{(2 M M)}$ occurs via triangle species composed of $L$ and $L^{*{ }^{*}}{ }_{(2 \mathrm{MM})}$ strands. Scale bars are $100 \mu \mathrm{m}$. Yellow frames indicate contrast enhanced images. Error bars in the graphs give standard deviation from four identical drops. Images of crystals show the image section at polarized white light illumination (WL) and fluorescence illumination (FL). 
a

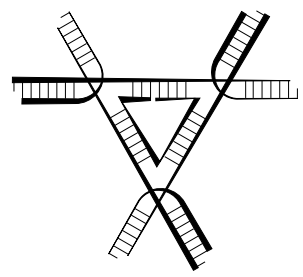

original b

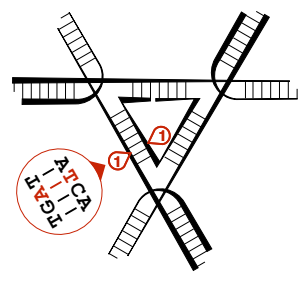

$\diamond$ core'$^{\prime}(+1 \mathrm{bp})$

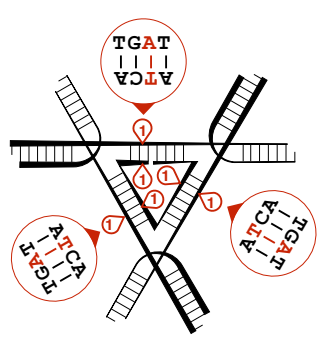

$\square$ core'(+3bp)

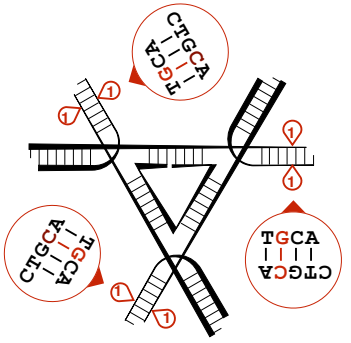

$\triangle$ arms'(+3bp) $^{\prime}$

C

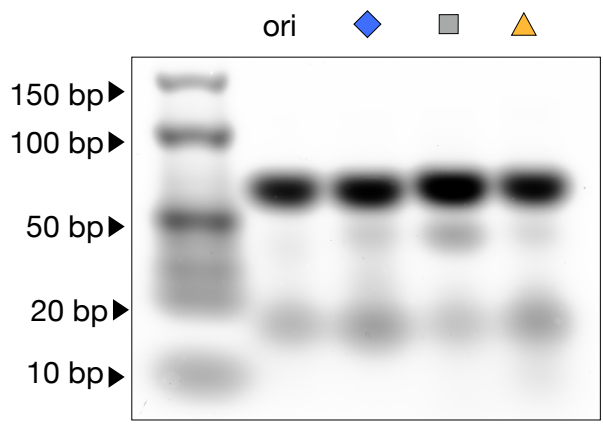

d

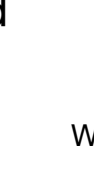
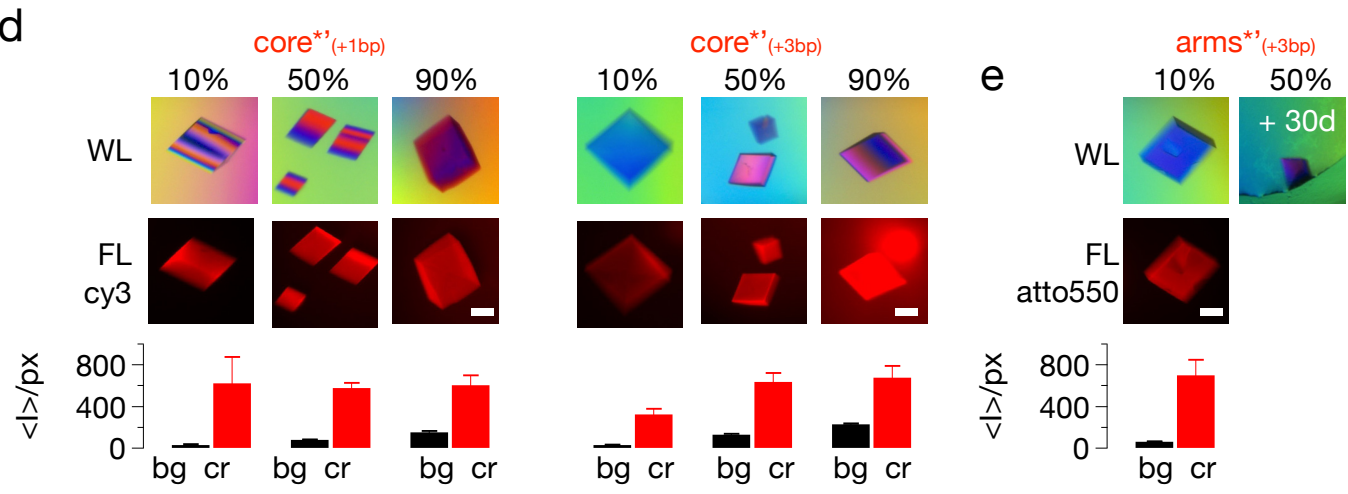

Figure S6. Analysis of triangle assembly and strand incorporation into the crystal lattice for strands that introduce a base pair insertion. $(\mathbf{a}, \mathbf{b})$ Schematics illustrate the composition of the original triangle design (a) and variations designed to contain base pair insertions in the core region (core') or in the arm region (arms') (b). (c) Gel electrophoretic analysis of variants in (a, b). Band pattern showed that all variants with base pair insertions assembled into triangles with a similar yield as the original strand composition. (d, e) Typical images of crystals from tracing experiments (WL, polarized white light illumination, FL, fluorescence mode) and quantification of the average fluorescence signal in the overall drops showed clear incorporation of all labelled and modified strands by significantly increased fluorescence intensity in the area covered by crystals (cr) as compared to the background area (bg). (e) In mixtures with $50 \% \mathrm{~S}^{*}$ ' and L' we only observed crystals in two out of four droplets after approx. 30 days of incubation, while crystals appeared after ten days for the same mixtures with unlabeled modified strands. Scale bars are $100 \mu \mathrm{m}$. Error bars in graphs give the standard deviation from four identical drops. Images of crystals show the image section at polarized white light illumination $(\mathrm{WL})$ and fluorescence illumination (FL). 
a

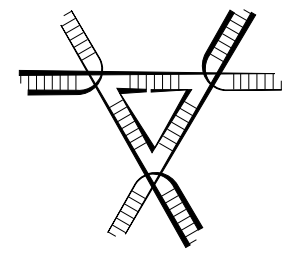

original b

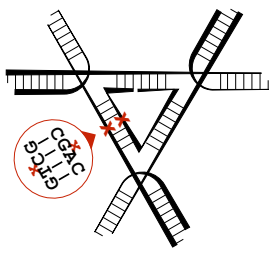

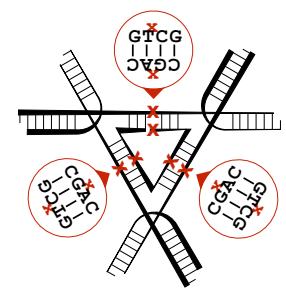

$\square$ core'(-3bp)

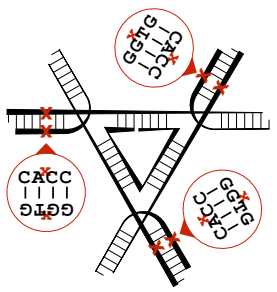

$\triangle$ arms'(-3bp)

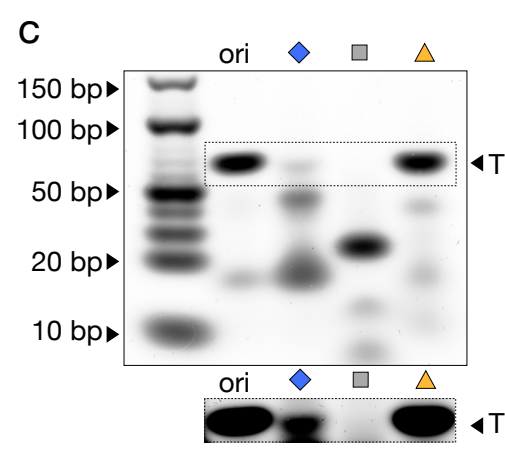

d

d
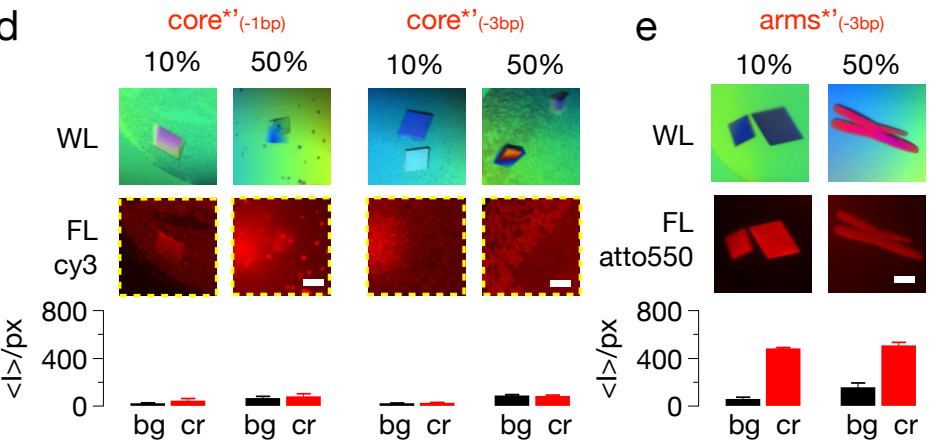

Figure S7. Analysis of triangle assembly and strand incorporation into the crystal lattice for strands that introduce a base pair deletion. (a, b) Schematics illustrate the composition of the original triangle design (a) and variations designed to contain base pair deletions in the core region (core' $_{(-1 \mathrm{bp})}$, core' $\left.{ }_{(-3 \mathrm{bp})}\right)$ or in the arm region of the triangle $\left(\mathrm{arms}_{(-3 \mathrm{bp})}\right)$. (c) Gel electrophoretic analysis of variants in $(a, b)$. Band pattern showed that base pair deletions in the core region significantly affected triangle assembly. Only the design variant core' ${ }_{(-1 \mathrm{bp})}$ managed to assemble into triangles, albeit with low yield. A base pair deletion in the arms region gave triangles with a similar assembly yield as seen for the original design (ori). The boxed gel area is shown again in a strongly contrast enhanced version below the gel. T indicates the triangle band. (d) Contrast enhanced fluorescence images (yellow frame) of typical crystals show faint incorporation of $\mathrm{M}^{\star}$ strands which contribute to formation of one basepair deletion in the core $\left(\right.$ core $\left.^{\left.*{ }_{(-1 b p}\right)}\right) . \mathrm{M}^{*}$ strands that contribute to the formation of three base pair deletions in the core (core $\left.{ }_{(-3 b p)}\right)$ are completely excluded from crystals, which are not even visible under fluorescence illumination (d, right images). Quantification of the overall fluorescence in the drops gave the same average fluorescence intensity for the area covered by crystals and for the background for both strand modifications. This can be attributed to the background behind and in front of the crystals. (e) Typical crystal images and fluorescence quantification showed incorporation of labeled $\mathrm{S}^{*}$ strands which contribute to the formation of a base pair deletion in the arm regions of the triangle (arms' ${ }_{(-3 \mathrm{bp})}$ ) by significant enrichment of average fluorescent intensity in the crystal area (cr) over the background signal (bg) can also be seen in drop quantification (bottom graph). The observed change in crystal habit from corresponding experiments with $50 \%$ modified strands was reproduced with labeled $L^{*}$-strands. Error bars give the standard deviation from four identical drops. Images of crystals show the image section at polarized white light illumination (WL) and fluorescence illumination (FL). Scale bars are $100 \mu \mathrm{m}$. 


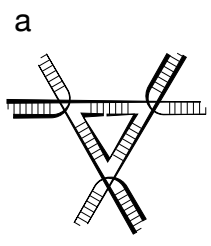

original b

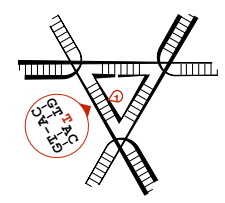

$\diamond \mathrm{M}^{\prime}($ (ns1)

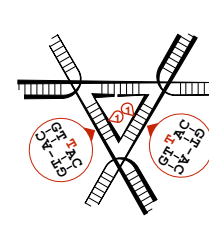

$\square \mathrm{M}^{\prime}($ (ns22x1)

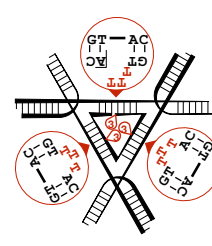

$\triangle M^{\prime}($ (ns3×3)

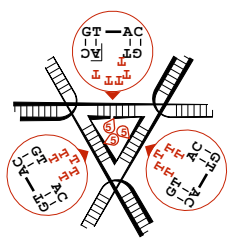

$\boldsymbol{\nabla} \mathrm{M}^{\prime}($ (ns $3 \times 5)$
C

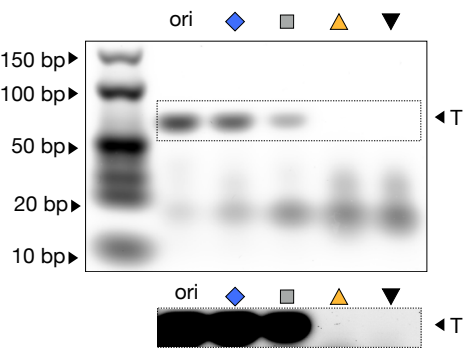

d

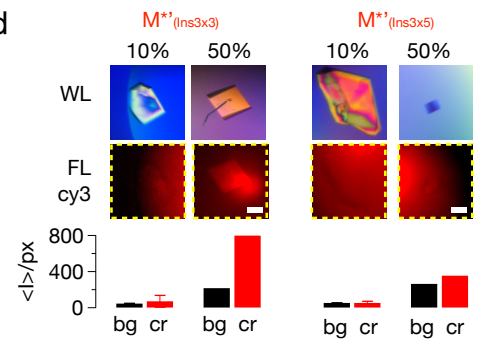

e
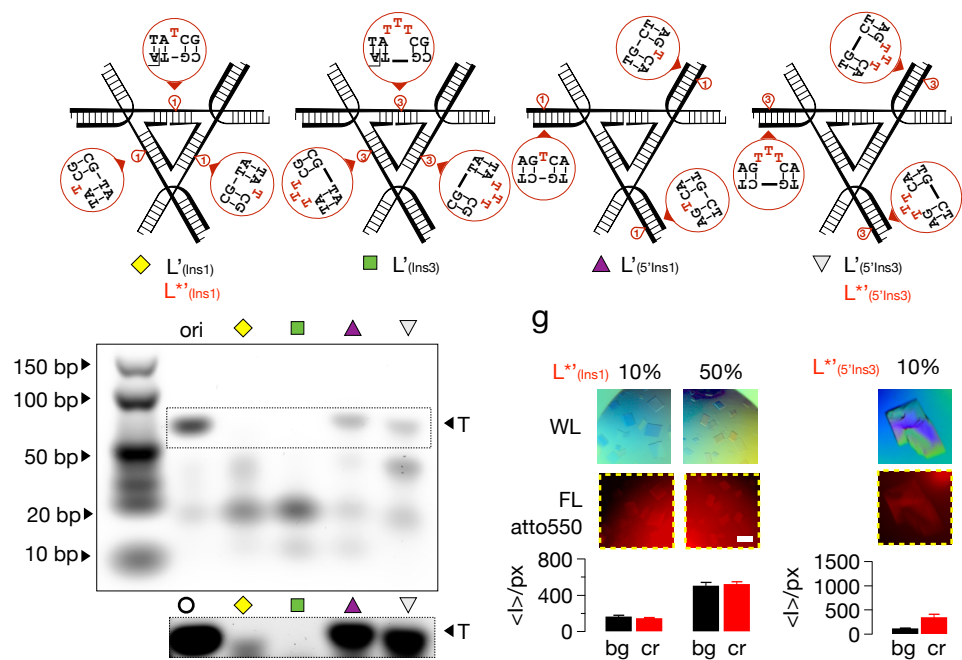

g
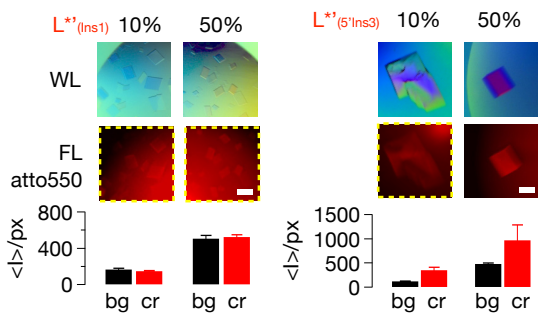

Figure S8. Analysis of triangle assembly and strand incorporation into the crystal lattice for strands that introduce insertion bulges. $(\mathbf{a}, \mathbf{b})$ Schematics illustrate the composition of the original triangle design (a) and variations designed to contain one-, three, and five-base bulges in the triangle core region introduced by the M-strand (b). (c) Gel electrophoretic analysis of variants in (a, b). Band pattern showed that triangles formed with one-base bulges $\left(\mathrm{M}_{(\text {(ns } 1)}{ }^{\prime}, \mathrm{M}_{\left({ }_{(n s 2 \times 1)}\right)}\right)$, but did not assemble with $\mathrm{M}$ strands introducing larger bulges $\left(\mathrm{M}_{(3 \times 3)}^{\prime}, \mathrm{M}_{(3 \times 5)}^{\prime}\right)$. The boxed gel area is shown again in a strongly contrast enhanced version below the gel. T indicates the triangle band (d) Fluorescence tracing gave faint incorporation of $\mathrm{M}^{*}$ strands with $3 \times 3$ insertions, as shown in strongly enhanced images of typical crystals, but fluorescence quantification indicates that the majority of $\mathrm{M}_{(3 \times 3)}^{*}$ strands stayed in solution (bottom graph). $\mathrm{M}^{*}$ strands with $3 \times 5$ insertions were not incorporated into the crystal lattice. (e) Schematics illustrate the composition of triangle variants designed to contain one- or three insertion bulges, introduced by a modified L strand. (f) Gel electrophoretic analysis of variants in (e). Band pattern showed that $L$ strands with insertions in the core region do not integrate into triangles. $L$ strands with insertions at the 5' region assemble into triangles, albeit with reduced yields. The boxed gel area is shown again in a strongly contrast enhanced version below the gel. T indicates the triangle band (f) Fluorescence tracing shows that $\mathrm{L}^{*{ }^{\prime}}{ }_{(\text {Ins1) }}$ strands are hardly integrated in the crystal lattice. Only strongly contrast enhanced images of crystals reveal a faint fluorescence of the crystals, but the average fluorescence intensity in the area covered by crystals (cr) is not higher as compared to the background area (bg) (bottom graph). $L^{*}{ }_{\left(5^{\prime} \prime n s 3\right)}$ strands are integrated into the crystal lattice, as shown by crystal images and fluorescence quantification of the drops. Scale bars are $100 \mu \mathrm{m}$. Error bars in graphs give the standard deviation from four identical drops. Images of crystals show the same image section at polarized white light illumination (WL) and fluorescence illumination (FL). (note) Also here, experiments with labeled $L^{*}$ strands show reduced inhibition of crystallization at $50 \%$ as compared to the same experiment with unlabeled $L$ strands. 
a

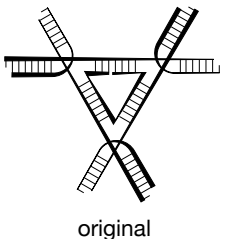

C

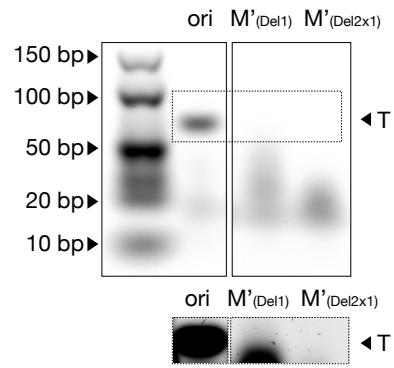

b

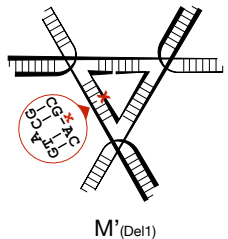

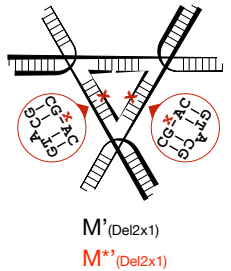

d

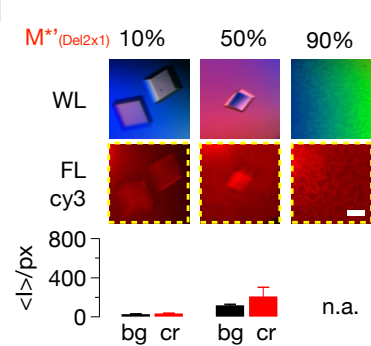

e
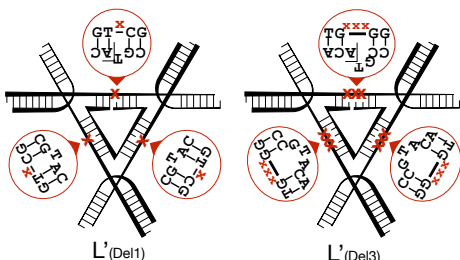

f
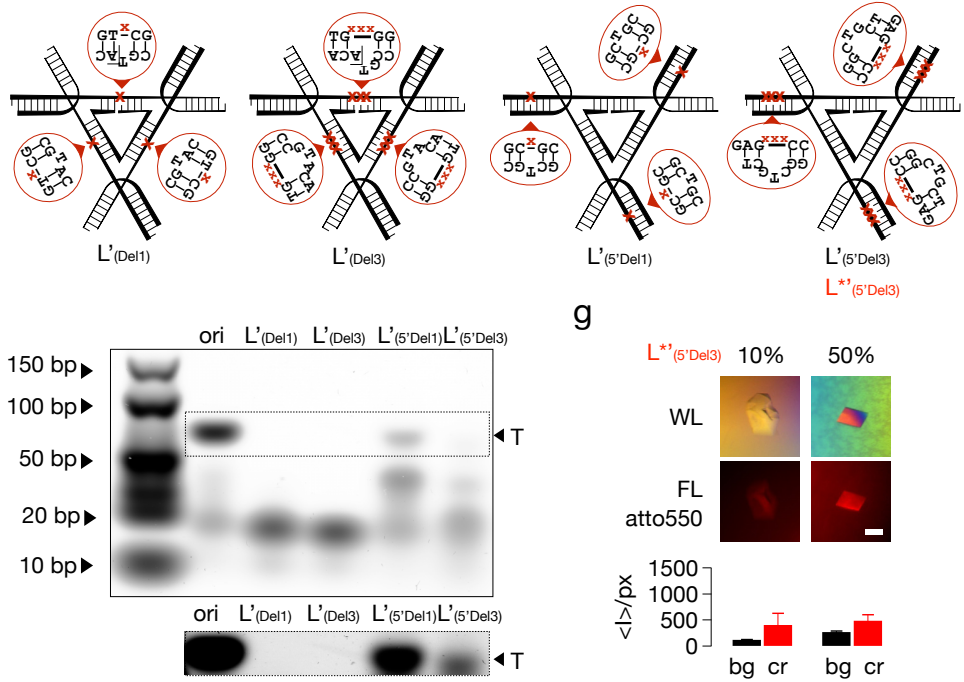

g

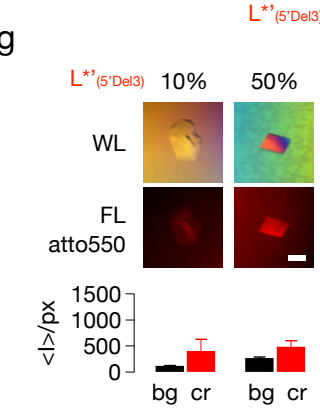

Figure S9. Analysis of triangle assembly and strand incorporation into the crystal lattice for strands that introduce deletion bulges. (a, b) Schematics illustrate the composition of the original triangle design (a) and variations designed to contain one-base bulges in the $L$ strands (created by deletions in the M strand) (c) Gel electrophoretic analysis of variants in (a, b). Band pattern showed poor assembly yield with $\mathrm{M}^{\prime}$ (Del1) strands, and no assembly with $\mathrm{M}_{(\mathrm{Del} 2)}$ strands. Image sections of the same gel are depicted to show the lanes with samples of interest. The boxed gel area is shown again in a strongly contrast enhanced version below the gel. $T$ indicates the triangle band (d) Fluorescence tracing with $\mathrm{M}^{*}{ }_{(\mathrm{Del} 2)}$ strands revealed that a small fraction of the labeled modified $\mathrm{M}$ strand was incorporated into the crystal lattice (yellow frames indicate contrast enhanced images). Instead of a second rod-like species - as observed for the same experiments with unlabeled strands - we observed the formation of precipitate with quenched fluorescence. (e) Schematic illustration of the triangle modifications designed to contain one and three-base bulges in the $\mathrm{M}$ or $\mathrm{S}$ strand (created by deletions in the $L$ strand). (f) Gel electrophoretic analysis of variants in (a, e). Band pattern showed that triangles did not assemble with deletions in the core region of the $L$ strand $\left(L^{\prime}{ }_{(D e \mid 1)}\right) L^{\prime}{ }^{\prime}$ Deli3) $)$, but weak assembly was observed with $L$ strands having deletions in the arm regions $\left(\mathrm{L}_{\left(5^{\prime} \mathrm{Del} 11\right)}, \mathrm{L}^{\prime}\left(5^{\prime} \mathrm{Del}\right) \mathrm{s}\right)$. The boxed gel area is shown again in a strongly contrast enhanced version below the gel. T indicates the triangle band. (g) Fluorescence tracing experiments gave weak integration of $L^{\prime}{ }_{\left(5^{\prime} \mathrm{Del}\right)}$ strands into the crystal lattice, as shown in fluorescence images of crystals $(F L)$ and from quantification of the overall fluorescence in four identical drops. Scale bars are $100 \mu \mathrm{m}$. Error bars in graphs give the standard deviation from four identical drops. Images of crystals show the same image section at polarized white light illumination $(\mathrm{WL})$ and fluorescence illumination (FL). (note) Also here, experiments with labeled $\mathrm{L}^{*}$ strands show reduced inhibition of crystallization at $50 \%$ as compared to the same experiment with unlabeled $L$ strands. 
a

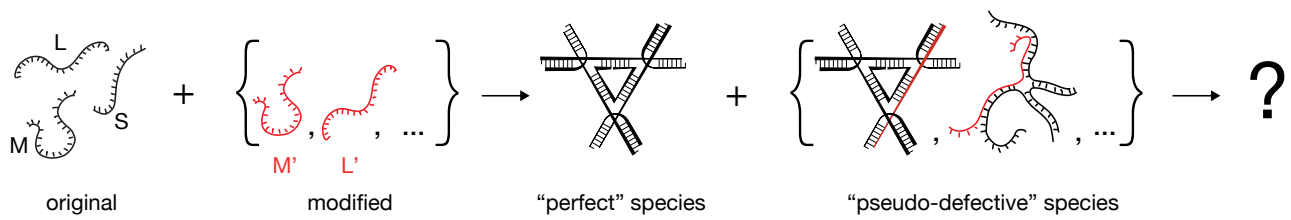

b

$\mathrm{mod} /(\bmod +$ original) $(\%)$

$\mathrm{mod} /(\bmod +$ original) $(\%)$
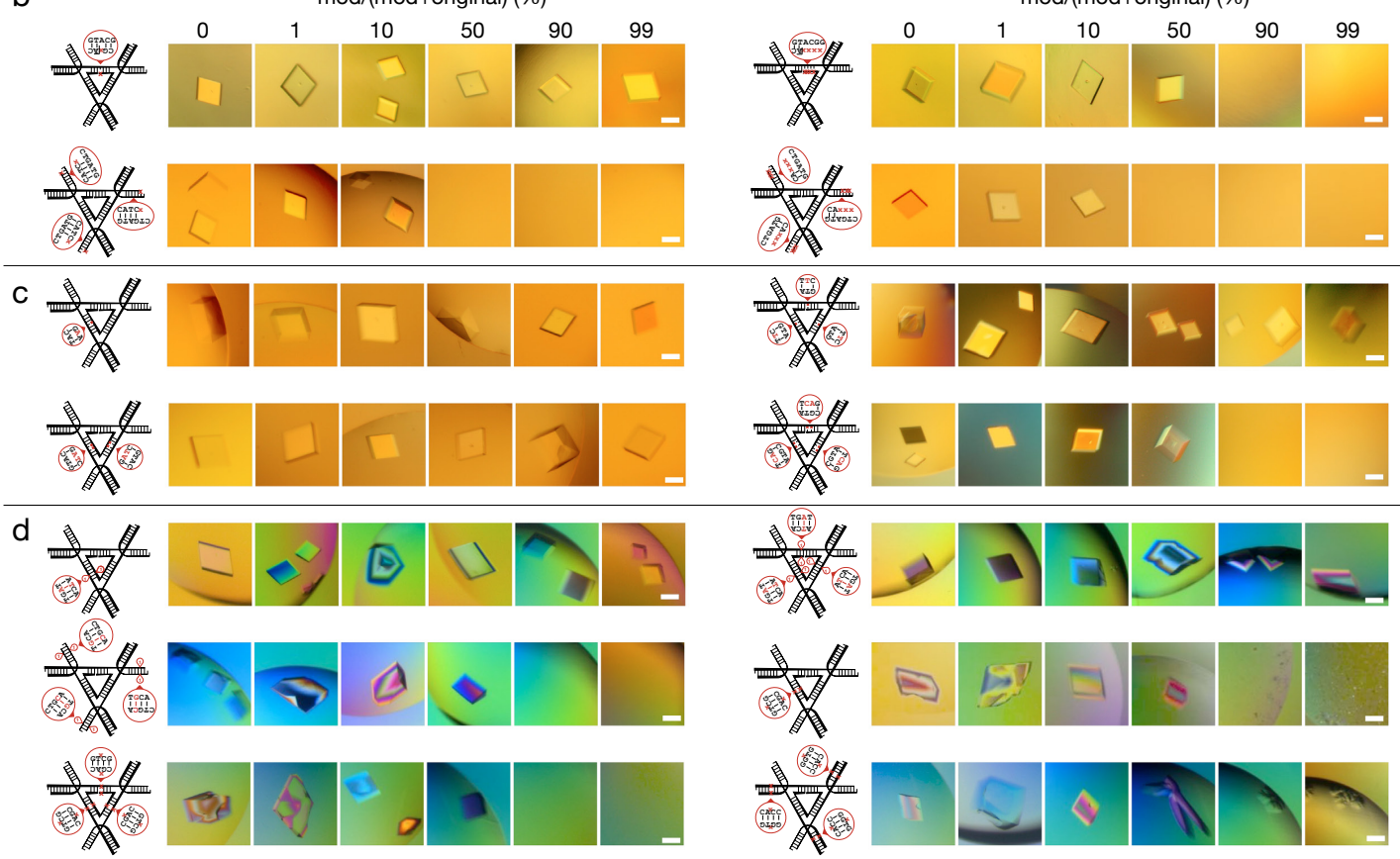

e
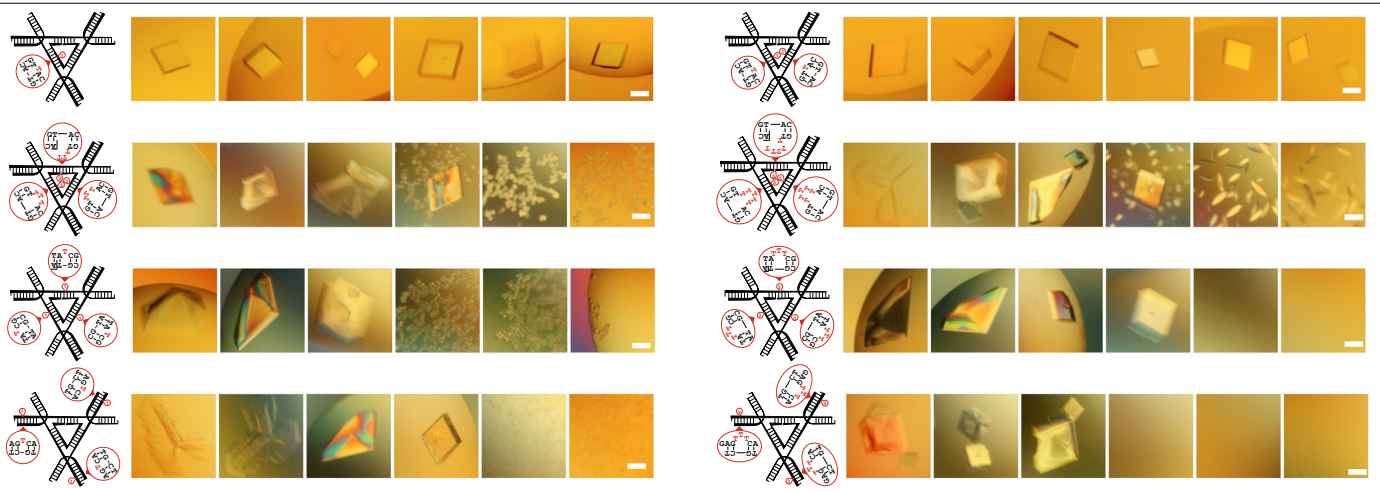

- 1
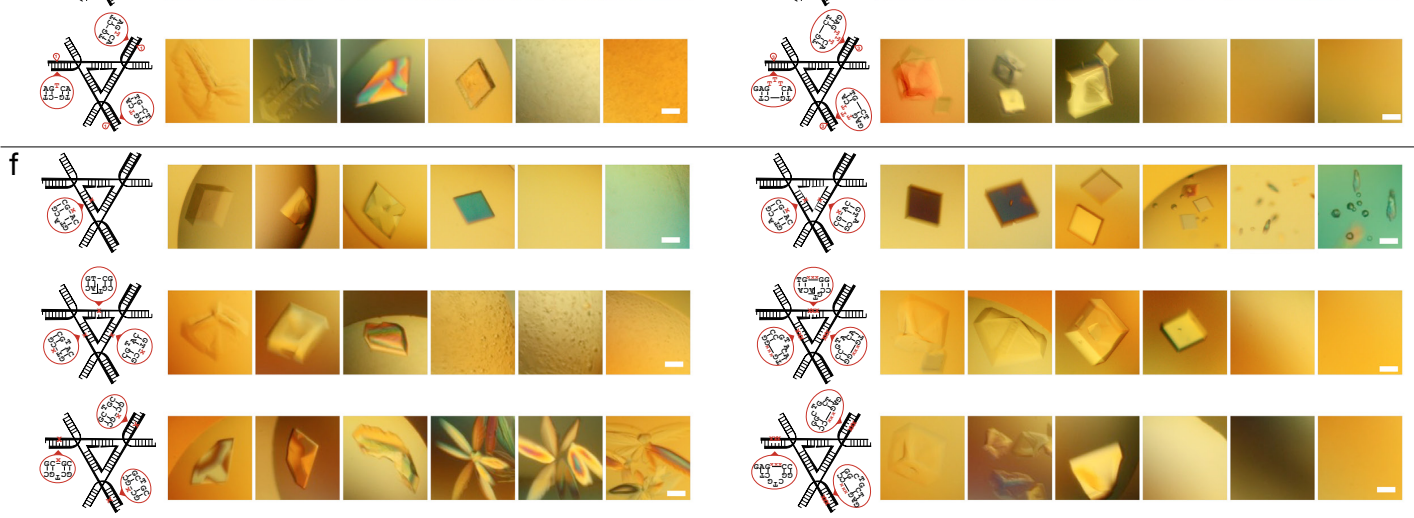

Figure S10. Extended version of Figure 2 showing all conditions tested in crystallization assays. (a) Specifically modified triangle strands (red) were added in addition to the default triangle strands to triangle crystallization droplets, to induce heterogeneity. (b-f) Typical images of crystals grown in presence of increasing content of modified strands (mod) and decreasing content of corresponding original strands (original). The total strand concentration was kept constant and the stoichiometry of triangle strands was maintained to meet a ratio of $3\left(L^{\prime}+L^{\prime}\right): / 1\left(M+M^{\prime}\right) / 3\left(S+S^{\prime}\right)$. Schematics give details of the triangle modifications to create truncations (b), sequence complementarity mismatches (c), base pair insertions and deletions (d), bulges induced by base insertions in one strand (e), and bulges induced by base deletions in the opposite strand (f). Scale bars are $100 \mu \mathrm{m}$. 


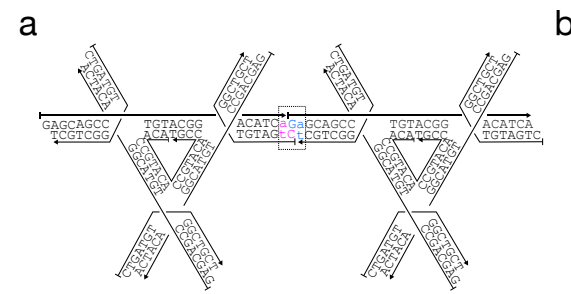

C
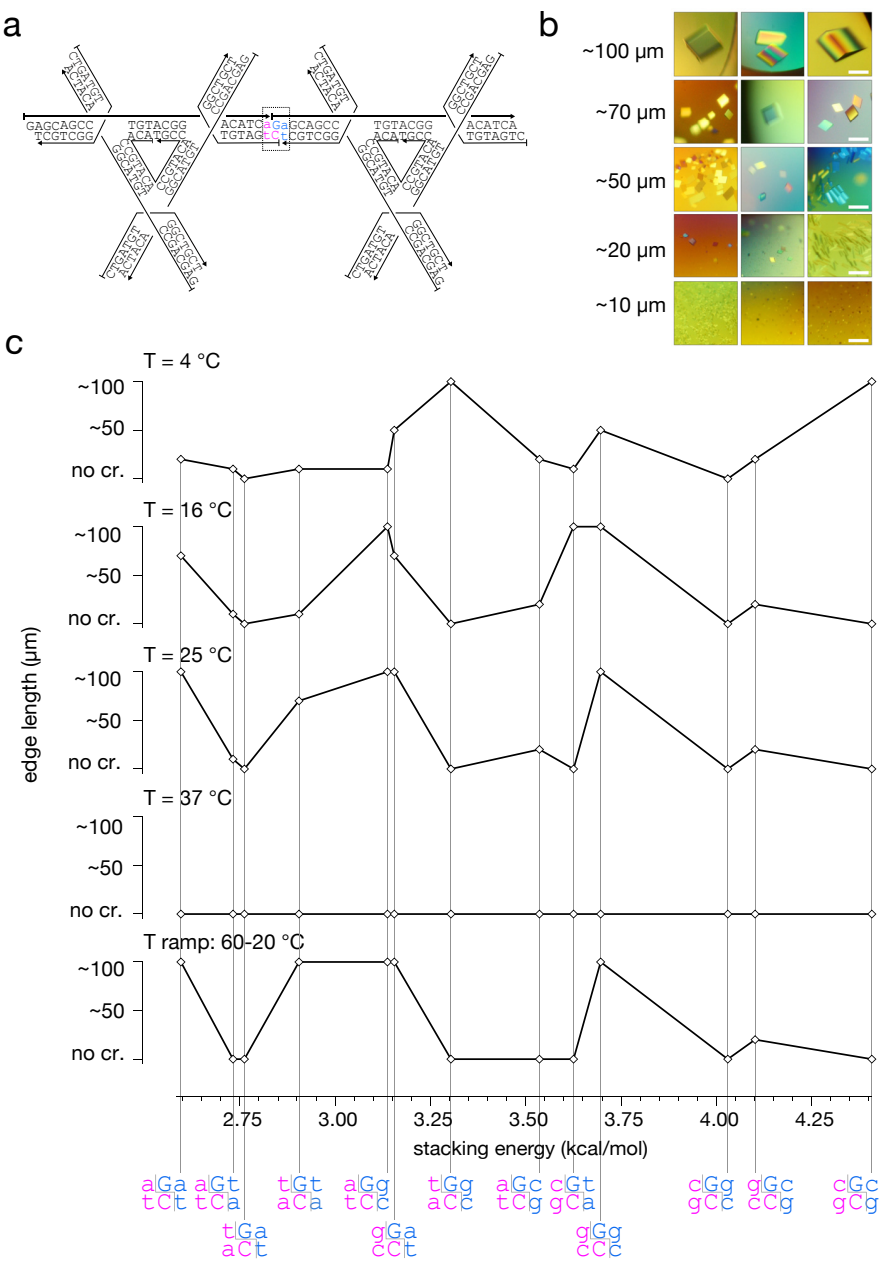

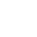

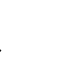


Table S1. List of oligonucleotide sequences. Modifications in pseudo-defective strands are highlighted in red for insertions and sequence variations. Regions where base were deleted are underlined.

original triangle strands
L GAGCAGCCTGTACGGACATCA
L* GAGCAGCCXGTACGGACATCA
X: amino-dT-atto550
M ACACCGTACACCGTACACCGT
$\mathrm{M}^{*}$ CY3-ACACCGTACACCGTACACCGT
S CTGATGTGGCTGCT
S* CTGATGTGGCXGCT
X: amino-dT-atto550

Figures 2 and 3: pseudo-defective triangle strands

truncations
$M^{\prime}\left(3^{\prime}-1\right)$ ACACCGTACACCGTACACCG_
$\mathrm{M}^{* 1}\left(3^{\prime}-1\right)$ CY3-ACACCGTACACCGTACACCG
$\mathrm{M}^{\prime}\left(3^{\prime}-4\right)$ ACACCGTACACCGTACA
$M^{* 1}\left(3^{\prime}-4\right)$ Cy3-ACACCGTACACCGTACA
L'(3'-1) GAGCAGCCTGTACGGACATC
L'(3'-3) GAGCAGCCTGTACGGACA
$L^{* 1}(3 '-3)$ GAGCAGCCXGTACGGACA
X: amino-dT-atto550

mismatches

M'(1MM) ACACCGAACACCGTACACCGT

$M^{\prime}(2 \times 2 M M)$ ACACCGATCACCGATCACCGT

L'(1MM) GAGCAGCCTGTTCGGACATCA

L'(2MM) GAGCAGCCTGTCAGGACATCA

\section{base pair insertions}
core' $^{\prime}(+1 \mathrm{bp})$
$\mathrm{M}^{\prime}(1 \times 8 \mathrm{ML})$ ACACCGTATCACCGTACACCGT
L'(8ML) GAGCAGCCTGATACGGACATCA
core $^{* 1}(+1 \mathrm{bp}) \quad \mathrm{M}^{* 1}(1 \times 8 \mathrm{ML})$ Cy3-ACACCGTATCACCGTACACCGT
L'(8ML) GAGCAGCCTGATACGGACATCA
core'(+3bp) $\quad \mathrm{M}^{\prime}(3 \times 8 \mathrm{ML})$ ATCACCGTATCACCGTATCACCGT
L'(8ML) GAGCAGCCTGATACGGACATCA
core*1(+3bp) M"'(3x8ML) Cy3-ATCACCGTATCACCGTATCACCGT
L'(8ML) GAGCAGCCTGATACGGACATCA
arms'(+3bp) $\quad \mathrm{S}^{\prime}(8 \mathrm{SL})$ CTGCATGTGGCTGCT
L'(8SL) GAGCAGCCTGTACGGACATGCA
$\begin{array}{ll}\operatorname{arms}^{* 1}(+3 b p) & S^{* 1}(8 S L) \text { CTGCATGTGGCXGCT } \\ & L^{\prime}(8 S L) \text { GAGCAGCCTGTACGGACATGCA }\end{array}$

X: amino-dT-atto550 


\section{base pair deletions}

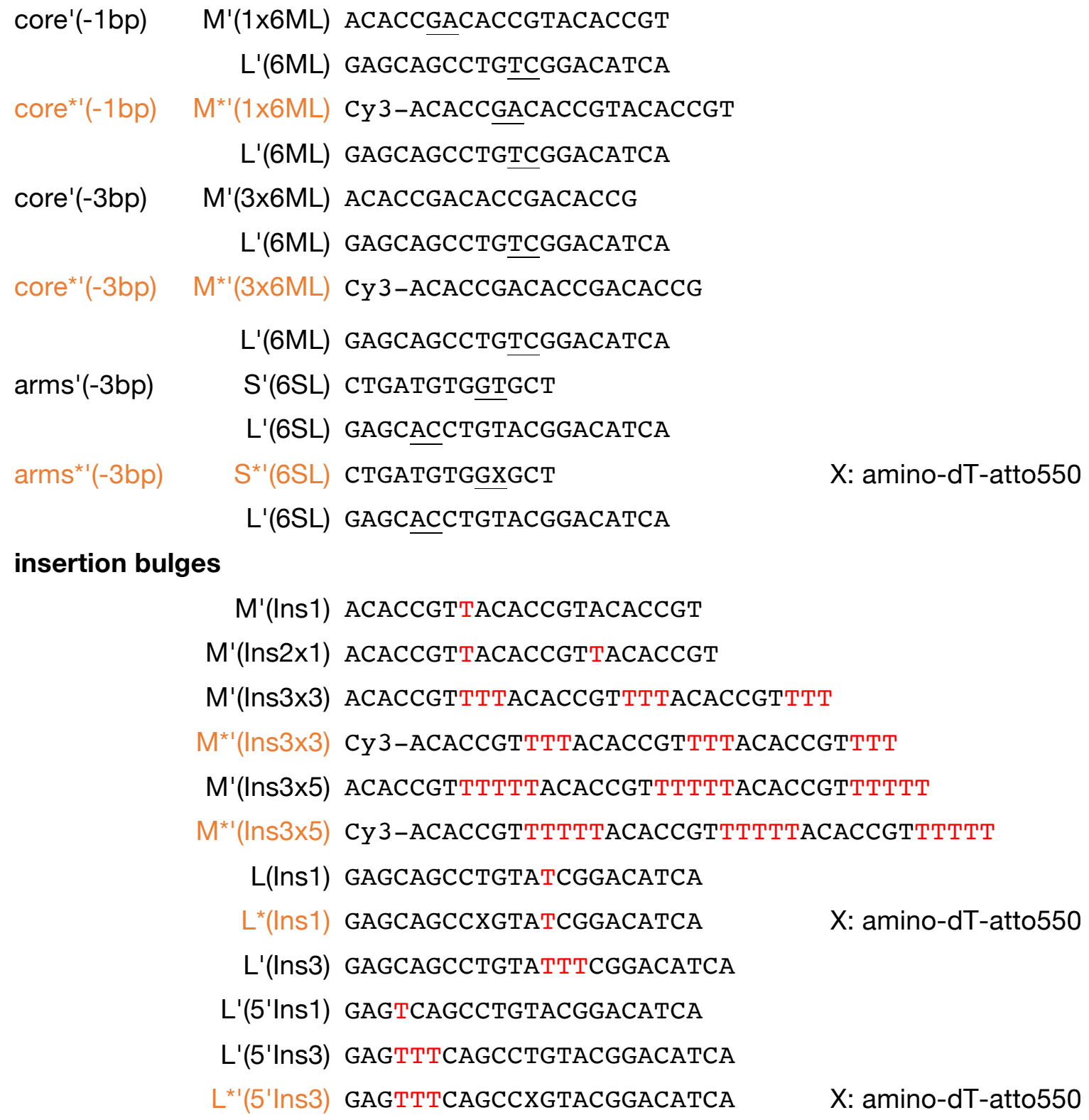

deletion bulges

M'(Del1) ACACCGACACCGTACACCGT

M'(Del2) ACACCGACACCGACACCGT

$M^{* 1}($ Del2) Cy3-ACACCGACACCGACACCGT

L'(Del1) GAGCAGCCTGTCGGACATCA

L'(Del3) GAGCAGCCTGGGACATCA

L'(5'Del1) GAGCGCCTGTACGGACATCA

L'(5'Del3) GAGCCTGTACGGACATCA

L*'(5'Del3) GAGCCXGTACGGACATCA X: amino-dT-atto550 
Figure 4f: sequence variation of the arm region

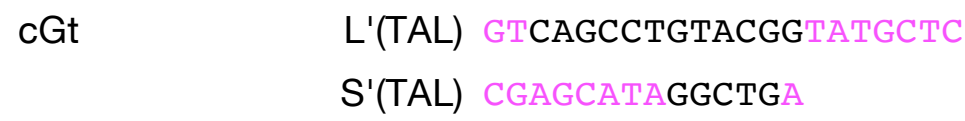

Figure 5: sequence variation of bases next to the GC-sticky end

\begin{tabular}{|c|c|c|}
\hline$c G c$ & $\mathrm{~L}(\mathrm{cGc})$ & GcGCAGCCTGTACGGACATCC \\
\hline & S8cGc) & CgGATGTGGCTGCg \\
\hline tGt & $L(t G t)$ & GtGCAGCCTGTACGGACATCt \\
\hline & $S(t G t)$ & CaGATGTGGCTGCa \\
\hline$g G g$ & $L(g G g)$ & GgGCAGCCTGTACGGACATCg \\
\hline & $S(g G g)$ & CCGATGTGGCTGCC \\
\hline gGa & $L(g G a)$ & GaGCAGCCTGTACGGACATCg \\
\hline & $S(g G a)$ & CCGATGTGGCTGCt \\
\hline cGt & $L(c G t)$ & GtGCAGCCTGTACGGACATCC \\
\hline & $S(c G t)$ & CgGATGTGGCTGCa \\
\hline $\mathrm{tGa}$ & $\mathrm{L}(\mathrm{tGa})$ & GaGCAGCCTGTACGGACATCt \\
\hline & $\mathrm{S}(\mathrm{tGa})$ & CaGATGTGGCTGCt \\
\hline $\mathrm{tGg}$ & $\mathrm{L}(\mathrm{tGg})$ & GgGCAGCCTGTACGGACATCt \\
\hline & $\mathrm{S}(\mathrm{tGa})$ & CaGATGTGGCTGCC \\
\hline cGg & L(cGg) & GgGCAGCCTGTACGGACATCC \\
\hline & $S(c G g)$ & CgGATGTGGCTGCC \\
\hline $\mathrm{aGc}$ & $L(a G c)$ & GcGCAGCCTGTACGGACATCa \\
\hline & $\mathrm{S}(\mathrm{aGc})$ & CtGATGTGGCTGCg \\
\hline aGg & L(aGg) & GgGCAGCCTGTACGGACATCa \\
\hline & $\mathrm{S}(\mathrm{aGg})$ & CtGATGTGGCTGCC \\
\hline aGt & $L(a G t)$ & GtGCAGCCTGTACGGACATCa \\
\hline & $S(a G t)$ & CtGATGTGGCTGCa \\
\hline gGc & $L(g G c)$ & GTACGGACATCg \\
\hline & $S(g G c)$ & CCGATGTGGCTGCg \\
\hline
\end{tabular}

TAL recognition site 


\section{REFERENCES}

1. J. Zheng, J. J. Birktoft, Y.Chen, T.Wang, R. Sha, P.E. Constantinou, S. Ginell, C. Mao, N.C. Seeman, From Molecular to Macroscopic via the Rational Design of a SelfAssembled 3D DNA Crystal. Nature 461, 74-77 (2009).

2. J. SantaLucia, Jr., D. Hicks, The Thermodynamics of DNA Structural Motifs. Annu. Rev. Biophys. Biomol. Struct. 33, 415-440 (2004).

3. H. T. Allawi, J. SantaLucia, Jr., Thermodynamics of Internal C.T Mismatches in DNA. Nucleic Acids Res. 26, 2694-2701 (1998).

4. H. T. Allawi, J. SantaLucia, Jr., NMR Solution Structure of a DNA Dodecamer Containing Single G.T Mismatches. Nucleic Acids Res. 26, 4925-4934 (1998).

5. K. L. Greene, R.L. Jones, Y. Li, H. Robinson, H.Wang, H.J.Andrew, and G. Zon, D.W. Wilson. Solution Structure of a GA Mismatch DNA Sequence, d(CCATGAATGG)2, Determined by 2D NMR and Structural Refinement Methods. Biochemistry 33, 10531062 (1994).

6. J. A. Rice, D. M. Crothers, DNA Bending by the Bulge Defect. Biochemistry 28, 45124516 (1989).

7. L. Joshua-Tor, F. Frolow, E. Apella, and H. Hope, Three-Dimensional Structures of Bulge-Containing DNA Fragments. J. Mol. Biol. 225, 397-431 (1992). 